Published in final edited form as:

Sci Signal. ; 2(82): mr5. doi:10.1126/scisignal.282mr5.

\title{
Chemical Approaches to Nuclear Receptors in Metabolism
}

\author{
Ronald N. Margolis ${ }^{1,{ }^{*}}$, David D. Moore ${ }^{2}$, Timothy M. Willson ${ }^{3}$, and R. Kip Guy ${ }^{4}$ \\ ${ }^{1}$ Division of Diabetes, Endocrinology, and Metabolic Diseases, National Institute of Diabetes and \\ Digestive and Kidney Diseases, National Institutes of Health, Bethesda, MD 20892, USA. \\ ${ }^{2}$ Department of Molecular and Cellular Biology, Baylor College of Medicine, Houston, TX 77030, \\ USA. \\ ${ }^{3}$ Department of Exploratory Chemistry, GlaxoSmithKline, Research Triangle Park, NC 27709, \\ USA. \\ ${ }^{4}$ Chemical Biology and Therapeutics Department, St. Jude Children's Research Hospital, \\ Memphis, TN 38105, USA.
}

\begin{abstract}
The National Institute of Diabetes and Digestive and Kidney Diseases (NIDDK) sponsored a workshop, "Chemical Approaches to Nuclear Receptors and Metabolism," in April 2009 to explore how chemical and molecular biology and physiology can be exploited to further our understanding of nuclear receptor structure, function, and role in disease. Signaling cascades involving nuclear receptors are more complex and interrelated than once thought. Nuclear receptors continue to be attractive targets for drug discovery. The overall goal of this workshop was to identify gaps in our understanding of the complexity of ligand activities and begin to address them by (i) increasing the collaboration of investigators from different disciplines, (ii) developing a better understanding of chemical modulation of nuclear receptor action, and (iii) identifying opportunities and roadblocks in the path of translating basic research to discovery of new therapeutics.
\end{abstract}

\section{Introduction and Background}

Nuclear receptors (NRs) are ligand-dependent and -independent transcription factors with major roles in development, organogenesis, reproduction, and metabolism (1). Their roles in disease have made NRs targets for the development of therapeutics for treatment of many diseases and disorders, including reproductive disorders, cancer, metabolic syndrome, cardiovascular disease, and diabetes. In the past decade, it has been recognized that the signaling cascades leading to NR action are complex and interrelated. Simultaneously, an understanding has arisen that the regulation of NR signaling is governed by more factors than ligand binding alone, with roles for multiple co-regulators, posttranscriptional and posttranslational modification, and other epigenetic processes.

*Corresponding author. rm76f@nih.gov.

A report on the workshop "Chemical Approaches to Nuclear Receptors and Metabolism," sponsored by National Institute of Diabetes and Digestive and Kidney Diseases, Bethesda, Maryland, USA, 16 to 17 April 2009. 
Our understanding of NR biology has benefited from pharmacological tools that allow manipulation of NR signaling, and although much has been done with existing tools, there is a clear need for additional tools with which to study NR structure and function. The role of chemical biology in fostering the development of methods and in identifying new tools for probing NR biology, perhaps leading to novel therapeutic approaches, presents an exciting opportunity for multidisciplinary research efforts. The goal of these collaborative efforts is to define how natural hormones, small synthetic molecules, and natural products may be used to uncouple complex hormone-induced events to provide selectivity and specificity with fewer off-target effects than agents that nonspecifically target entire pathways. The specific focus of the workshop was diseases and disorders associated with lipid and carbohydrate metabolism, obesity, and type 2 diabetes (T2D).

NR biology has been a rich area for drug discovery: Glucocorticoids for asthma, retinoids for acne, and estrogens for hormone replacement therapy are but a few. All of these drugs were developed before the advent of molecular biology. Despite many scientific breakthroughs in the basic biology of NR signaling, these have yet to successfully translate into an approved drug that can give the desired specificity and selectivity of action without off-target effects. In the field of NR drug discovery, industrial research has begun to focus its efforts on late therapeutic discovery and early preclinical research, whereas academic investment is concentrated in basic discovery research. The result is that the intermediate steps in translating basic research to applications at the bedside suffer from a lack of investment from either academia or industry (Fig. 1). Through the Molecular Libraries project, the NIH Roadmap has sought to bridge the gap between basic discovery and translation by introducing chemical biology as an academic discipline (2). The field of chemical genomics, the study of how small molecules interact with targets in cells, has thus emerged, and the NIH has provided resources to individual investigators and collaborative teams for the development of specific chemical probes for research applications and preclinical therapeutic discovery (www.mli.nih.gov). In addition, global projects, such as the Nuclear Receptor Signaling Atlas (www.nursa.org), cosponsored by several NIH institutes (NIDDK; National Heart, Lung, and Blood Institute; National Institute of Environmental Health Sciences), seek to coalesce information about NRs, co-regulators, ligands, and their roles in disease in central and easily accessible data repositories (3). Together, these resources provide powerful tools for investigators seeking to better develop approaches for probing NR action.

\section{Evolution of NR Specificity}

NRs are highly evolutionarily conserved, and Joe Thornton (University of Oregon, Eugene) described how a common ancestor is hypothesized to have given rise to the 49 human receptors through duplication and subsequent divergence (4). By reconstructing the ancestral steroid receptor gene, Thornton determined how the earliest receptor precursor specific to one steroid hormone led ultimately to the functional diversity that we see today. Nuclear receptors are present in various forms in almost all animals, with an ancestral corticoid receptor arising $\sim 420$ million years ago. This receptor had as its ligand an ancient hormone with mineralocorticoid-like activity and was likely associated with maintaining proper ion balances. Through receptor duplication and exploitation of newly emerging ligands, this 
ancient corticoid receptor adopted new functions (4). For example, the glucocorticoid receptor (GR) likely evolved by loss of sensitivity to the ancient mineralocorticoid hormone. In the lineage extending from the elasmobranchs (skates and rays) to the teleosts (ray-finned boney fishes), GR specificity and sensitivity to cortisol were acquired through many mutations, particularly those affecting the ligand pocket. In this case, it would appear that the receptor preceded the ligand. As new variations of corticosteroids appeared as a result of mutations in cytochrome p450 enzymes, some of these novel ligands, such as cortisol, were able to bind to these GR variants, thus providing evolutionary potential for the elaboration of additional receptor-ligand partnerships. The overall concept is one of epistasis in that permissive mutations ultimately lead to a shift in ligand preference. The conclusion is that receptors are only as specific as they must be in order to exclude endogenous steroids and enable a specific response.

Another very ancient receptor is the estrogen receptor (ER) (5), and John Katzenellenbogen (University of Illinois, Urbana) pointed out that compounds of diverse structure having a phenolic back-bone can function as estrogens (6). Although many compounds can activate ERs, tissue selectivity is conferred by tissue-specific activation of different effector gene networks. Given the prominent role that the ER plays in development, reproduction, and metabolism, it is important to ask how estrogens can mediate so many different processes and still maintain tissue selectivity. And why do so many compounds have estrogen-like activity? The answers may lie in the universe of coactivators in a given cell, with liganddependent recruitment of co-regulators contributing to the specific response. The ligand binding pocket is another important determinant of signaling specificity, and how a ligand binds to a receptor influences the downstream responses. Structural studies of the ligand binding pocket, found in all but a few of the orphan nuclear receptors, and of the DNA binding and dimerization domains are critical to understanding how signaling specificity is generated.

\section{The Ligand Binding Domain}

Many advances in understanding of ligand-receptor interactions have stemmed from the application of structural biology approaches to the ligand binding domain (LBD) of the NR, first solved for thyroid receptor (TR) by John Baxter and co-workers (7). Recently, Fraydoon Rastinejad (University of Virginia, Charlottesville) has taken the study of NRs to a new level by solving the $\mathrm{x}$-ray structure of the PPAR-RXR (peroxisome proliferator-activated receptor-retinoid X receptor) heterodimer bound to DNA (8). Through this work, his group has demonstrated how each region of the receptor contributes to facilitating and/or maintaining dimerization and binding to coactivators and DNA. Structural analyses such as this have become important paradigms through which the chemical biology approach to receptor function is informed. Ruben Abagyan (Scripps Research Institute, La Jolla, California) further defined the concept of structural chemodynamics, in which molecular simulations of ligand binding are used to assay the effects of mutations at key residues on ligand binding (9), for the analysis and subsequent profiling of ligand-receptor interactions. Abagyan defined the "pocketome" of each receptor as distinct functional states, a collection of conformations determined by the shape the pocket takes upon binding to a given ligand. Though the LBD was once thought to be of fixed size and specific to a given receptor, it now 
appears that the ligand itself can play an important role in shaping the pocket to fit the ligand. In so doing, the receptor is "bent" in different ways, thus enabling differential cofactor recruitment and gene expression regulation. Thus, induced fit by a ligand upon entry into the ligand pocket gives rise to changes in the structure of the receptor through induction of multiple receptor conformations, the "NR pocketome."

One goal of understanding induced fit is to identify new ligands with which to alter the structure of the pocket and thus the function of the receptor, such as the androgen receptor (AR) and its response to antagonists and partial agonists (9). Extension of such analyses to orphan nuclear receptors (ONRs), which are nuclear receptors for which no definitive natural ligand has been identified, may help to "deorphanize" them. John Koh (University of Delaware, Newark) further showed how mutant NRs with mutations in the LBD can be chemically rescued by providing ligands capable of binding the mutant pocket (10). In disorders such as vitamin D-dependent rickets, resistance to thyroid hormone, and thyroid cancer, compounds that bind mutated NRs can overcome the effects of the mutation. Similarly, Tom Scanlan (Oregon Health Sciences University, Portland, Oregon) described how he has utilized the crystal structure of the active form of thyroid hormone (T3) bound to the LBD of TRa to show that T3 binds to both the $a$ and $\beta$ isoforms or TR, but compounds can be developed (GC-1, or sobetirome, for example) that selectively bind to only one isoform, thus providing the requisite selectivity to treat disease (11). Keith Yamamoto (University of California, San Francisco) discussed how binding of the GR receptor to the glucocorticoid response element (GRE), in turn, affects the shape of the receptor (12), suggesting that variants of consensus hormone response elements such as glucocorticoid binding sequences (GBSs) may allosterically modify the receptor and thus alter its ability to regulate expression of target genes. Integrating the effect of both ligand and GRE binding at the GBS may therefore be crucial to differential utilization of functional GR surfaces.

\section{Development and Use of New Tools}

The development and application of new tools and technological approaches have had a profound effect on the elucidation of ligand-receptor interactions and function. By exploiting the established genetic methods and known genomic sequences of model organisms, Henry Krause (University of Toronto) demonstrated how NR responses to ligands for the regulation of basic physiology may lead to new insights (13). As an example, dysregulation of metabolic pathways in fruit flies, such as disruption of circadian periodicity, leads to uncontrolled feeding and obesity. Such findings may provide new mechanistic insight into mammalian diseases. Pat Griffin (Scripps Research Institute, Jupiter, Florida) demonstrated how small-molecule compounds can be developed from high-throughput screening assays to provide tools for selectivity profiling and pathway discovery (14). Groups such as these are collaborating in a large NIH-funded effort, the Molecular Libraries Production Centers Network (MLPCN), to provide assay development, high-throughput screening, and synthetic and medicinal chemistry capabilities (www.mli.nih.gov). For the ONR SF-1, this led to discovery of an inverse agonist that has proven useful in further elucidating SF-1 function. Paul Johnston, who is also using small-molecule libraries in cell-based high-content screens, showed that ligand-induced nuclear translocation of the GR required dynein-mediated transport. In addition, protein-protein interaction assays showed how AR-coactivator 
complexes can identify compounds that interfere with the receptor-coactivator interface and subsequently modulate the proliferation of prostate tumors. Anna Mapp (University of Michigan, Ann Arbor) described small molecules capable of acting as transcriptional switches that they used to study the activation factor 1 (amphipathic or N-terminal transcription domains) of NRs (15). These domains are difficult to characterize and may hold the key to understanding the complexity and diversity of transcriptional activities attributed to NRs.

\section{Posttranscriptional Modification of NRs and Co-Regulators}

Bert O'Malley (Baylor College of Medicine, Houston, Texas) has pioneered the study of transcription factor co-regulators, including the p160 family of NR coactivators, members of which have been identified as potential therapeutic targets, including SRC-1 and -3 in cancer and SRC-2 in blood glucose regulation (von Gierke's disease) (16). Co-regulators appear to constitute a set of metabolic or "master" genes that encode proteins of various forms and perform a range of functions. The combinatorial nature of coactivator complexes implies that they may thus contribute to polygenic disease (T2D, for example). Notably, coactivators are targets for posttranscriptional modification (PTM), including phosphorylation, acetylation, ubiquitination, and sumoylation. Modification of co-regulator activity or cellular localization can have a profound effect on NR-mediated gene expression. Holly Ingraham (University of California, San Francisco) provided a specific example of how sumoylation, a reversible modification that occurs at specific consensus sequences within target proteins, may be a powerful means of regulating transcriptional activation (17). Among the NRs, only the GCNF, EAR2, and SHP ONRs lack this site. Ingraham demonstrated that SUMOylation represses SF-1, by changing its localization or stability, and altering the SUMO marks on SF-1 affects adrenal and gonadal morphology during development. Furthermore, combinatorial PTM may play a role, because phosphorylation of SF-1 may facilitate its subsequent SUMOylation.

Richard Pestell (Thomas Jefferson University) showed that co-regulators such as the p300 histone acetyltransferase may modify both NRs and other co-regulatory proteins (18). Acetylation of ERa at two sites determined the receptor's ligand sensitivity and affected its ability to bind to chromatin. Cultured cells harboring mutant ARs that showed altered acetylation displayed increased contact-independent growth and AR recruitment to chromatin, in response to ligands. Conversely, the class III histone deacetylase Sirt1 deacetylated $\mathrm{p} 300$, which decreased AR signaling and blocked growth in cultured prostate cancer cells. Thus, regulation of either the activity of the co-regulator or its PTM may serve as an important new chemical approach for modulating NR function.

\section{Nontraditional Chemical Approaches}

Antonio Bianco (University of Miami) presented one of several late-breaking stories selected from poster abstracts. Enzymes of the deiodinase family (DIO1-3) convert the inactive form of thyroid hormone $\mathrm{T} 4$ into the active form $\mathrm{T} 3$ or to the inactive form reverse T3, also called rT3 (19). Thus, chemical modification of a ligand within the cell plays a crucial role in determining the amount of active hormone present at a given time. Large 
amounts of T3 can result in increased oxygen consumption, whereas insufficient quantities of T3 may lead to loss of signaling at a critical time in cell differentiation. The DIO family of enzymes may therefore represent a novel therapeutic target for regulating cell metabolism. Bruce Spiegelman (Dana-Farber Cancer Institute, Boston, MA) showed how chemical biology could be applied to the PPAR $\gamma$ coactivator 1 (PGC-1) class of coactivators (20). PGC-1 $\alpha$ and PGC-1 $\beta$ have broad effects in many tissues and cells because they play a critical role in the regulation of energy balance. Expression of both PGC-1 isoforms is inducible and their activity is amenable to chemical manipulation; thus, they are candidate targets for therapeutic manipulation. Chemical modulators of PGC-1 activity may be useful for disorders of energy balance, such as T2D or denervation-induced muscle atrophy in which oxidative phosphorylation is reduced. Preliminary screens drawing from smallcompound collections have already yielded promising leads on compounds that may be used to modulate PGC-1 activity.

Continuing with the theme of innovative chemical approaches to manipulating nuclear receptor signaling, Vamsi Mootha (Harvard Medical School and the Broad Institute, Cambridge, MA) focused on chemical biology as a means to explore mitochondrial function (21). Both the mitochondrial and nuclear genomes (which encode 13 and 1200 proteins, respectively) contribute to mitochondrial function, making a genetic approach to studying this organelle problematic. Mootha's chemical approach, using a high-throughput assay for cell viability, mitochondrial physiology, and gene expression, was designed to systematically understand the structure and physiology of mitochondria and has led to better understanding of oxidative phosphrylation (oxphos) reactions (21). His goal is to exploit basic understanding of mitochondrial biology and its role in drug metabolism and toxicity to develop novel therapeutics to coordinate metabolic processes controlled jointly by the mitochondrial and nuclear genomes. Because many NRs, including the PPAR $\delta$, play key roles in mitochondrial oxphos reactions and may be affected by changes in reactive oxygen species (ROS), it may be clinically useful to develop compounds to increase mitochondrial oxphos while simultaneously decreasing ROS. Donald McDonnell (Duke University) has used phage display to identify specific regions of the AR that are required for differential recruitment of coactivators (22). Application of profiling technologies may aid in identifying compounds that affect NR transcriptional activity in cancer or metabolism, either decreasing proliferative signaling or modulating signals that affect oxphos.

\section{Large-Scale Data Handling}

Many genomic studies to identify functional roles for NRs in disease generate large amounts of data, the integration and analysis of which can be challenging. Shankar Subramaniam (University of California, San Diego) demonstrated that data from large studies of gene expression patterns could be assembled into networks by analyzing individual predicted regulatory interactions (23). This method was extended to clinical studies of patients treated with thiazoladinediones (TZDs, which are PPAR $\gamma$ agonists used to treat diabetes), and the results were analyzed by a variety of algorithms, many of which are freely available opensource computational programs, such as VAMPIRE (http://sasquatch.ucsd.edu/vampire/). Results can be analyzed by tissue type (such as skeletal muscle versus macrophage), class of genes (those that encode proteins involved in oxphos reactions, for example), or response to 
a drug such as the TZD rosiglita-zone. Assembling such specific networks from complex data sets may enable the development of new hypotheses or insights into human responses to drugs, inspire additional studies on other cellular pathways, or identify downstream targets for the development of new therapeutics.

\section{Translational Research}

Erin Schuetz (St. Jude's Children's Research Hospital) discussed a putative role for activators of the Pregnane X Receptor, a xenobiotic receptor, in the development of nonalcoholic fatty liver disease (NAFLD) (24). Constitutively activated PXR mimics the effects of human chronic drug administration, such as that which occurs with rifampicin treatment, and leads to hepatic steatosis. NAFLD is characterized by excess triglyceride accumulation and insulin resistance, and humanized mouse models (transgenic mice expressing a $h P X R$ transgene) are used to test the effects of NR ligands in vivo under conditions that mimic the human disease. Questions raised from such studies included the role of diurnal variation in development of fatty liver phenotype, as well as whether circadian periodicity in the abundance of drug-metabolizing enzymes could affect the amount of circulating drug and thus should dictate the timing for therapeutic drug administration. Beatrice Desvergne (University of Lausanne, Switzerland) discussed the emerging problem of endocrine disruption resulting from exposure to low concentrations of NR ligands present in the environment such as pthalates, which are commonly found in most plastics (25). Endocrine disruptors have been linked to effects on PPARs, possibly contributing to obesity through differential recruitment of coactivators, thus leading to alterations in expression of downstream effector genes. Desvergne reported that many such environmental compounds induced peroxisome proliferation in rodents, at least partly through acting as agonists for the PPARs.

David Moore (Baylor College of Medicine) discussed novel ligands that act through NRs with heretofore underappreciated roles in diabetes (26). Indeed, there is tantalizing evidence that ligands of many NRs might be used to treat diabetes (PPARs, ERa, CAR, TR $\beta$, LRH-1, FXR, LXRa/ $/$ ). Moore cited the example of LRH-1, an ONR that has emerged as a potential target for treatment of metabolic diseases. LRH-1 has been shown to copurify and co-crystallize with various phospholipids, but there is controversy over whether phospholipids function as physiological ligands for NRs or simply stabilize the aporeceptor. Moore noted that phosphatidylcholine constitutes $10 \%$ of the volume of the nucleus and described the ability of unusual phosphatidylcholine species with relatively short fatty acid acyl side chains to potently activate LRH-1. Administering these compounds to mice induced expression of genes that are repressed in the liver-specific LHR-1 knockout mice and modestly increased bile acid pools. This treatment was also associated with a marked improvement in insulin sensitivity in mouse models of T2D, suggesting LHR-1 as a potential therapeutic target. John Baxter (Methodist Hospital, Houston, Texas) demonstrated that some TR $\beta$ agonists lower circulating low-density lipoprotein without affecting heart rate, whereas TRa agonists increased heart rate and were thus limited in their clinical utility. One such TR $\beta$-selective compound, GC-1 (also called sobetirome), was developed as a possible treatment for obesity and T2D (27). 
Finally, Tim Willson (GlaxoSmithKline, Research Triangle Park, North Carolina) discussed an open-access listing of chemical probes for regulation of gene transcription. The pharmaceutical pipeline provides less than 10 innovative new drugs ("new chemical entities" or NCEs) per year, more than $90 \%$ of which fail in human trials due to lack of efficacy. More high-quality in vitro and in vivo target validation is needed to increase the number and quality of drug candidate compounds in the pipeline. New small-molecule chemical probes are important for identifying and validating new targets, as well as for mechanistic studies. Public-private partnerships may help to identify and disseminate such probes.

GlaxoSmithKline (GSK) has produced probes for many ONRs that are available through commercial suppliers such as Sigma-Aldrich. Modeled on this open-access philosophy, GSK has created a partnership with the Structural Genomics Consortium, Wellcome Trust, and NIH's MLPCN to discover and distribute chemical probes for epigenetic regulators of transcription. Probes can be used for target validation and to further our scientific understanding of the role of transcriptional regulation in disease. As more probes emerge from industry sources, such as GSK and the NIH-funded, MLPCN, effort, probe reports will be placed in a public repository at the National Library of Medicine (www.ncbi.nih.gov/ bookshelf) Other public repositories of relevant information include the Nuclear Receptor Signaling Atlas (www.nursa.org), an NIH-funded resource for information on NRs, coregulators, and ligands. Expansion of these and other online repositories will greatly enhance the pace of discovery and development.

\section{Summary and Future Directions}

A major theme that emerged from the workshop is that the ligand binding pocket is not a fixed structure, and the observation that its shape is modified by the cognate ligand suggests that synthetic ligands can be developed to have different effects on receptor activity and tissue selectivity. R. Kip Guy (St. Jude's Children's Research Hospital) closed the workshop by identifying key questions stemming from this theme, including: What response elements modulate which genes? How are phenotypes at both the tissue and organism level regulated by cellular NR function? What role does the cellular balance between ligand availability, coregulators, PTM, and chromatin state play in the regulation of target genes? Developing a better understanding of ligand-receptor interactions involves integration of multiple levels of disparate data into testable models. From such models come important questions for translational research: Can we target co-regulators? Can NRs be reliably modulated in "noncanonical" ways? How can we develop assays that will contribute to identification of ligands with better specificity and selectivity than endogenous or currently available synthetic ligands?

To answer many of these questions, it will be necessary to expand our current toolkit by developing novel chemical modulators and identifying which of these represent viable preclinical or clinical candidates, using them to better define structure-function relationships, and possibly using them to modulate mutant receptors, specific receptor isoforms, and coregulators. Chemical and molecular studies must be accompanied by development of computational approaches to modeling the NR pocketome and higher-order transcriptional complexes. Ultimately many of these approaches and applications of new tools may lead to better profiling of NR structure and function that gives rise to a more complete 
understanding of the integrated physiology of these important signaling molecules. Chemists and biologists working across disciplines will be required to address these important scientific questions.

\section{References}

1. Mangelsdorf DJ, Thummel C, Beato M, Herrlich P, Schütz G, Umesono K, Blumberg B, Kastner P, Mark M, Chambon P, Evans RM. The nuclear receptor superfamily: The second decade. Cell. 1995; 83:835-839. [PubMed: 8521507]

2. Austin CP, Brady LS, Insel TR, Collins FS. NIH Molecular Libraries Initiative. Science. 2004; 306:1138-1139. [PubMed: 15542455]

3. McKenna NJ, Cooney AJ, Demayo FJ, Downes M, Glass CK, Lanz RB, Lazar MA, Mangelsdorf DJ, Moore DD, Qin J, Steffen DL, Tsai MJ, Tsai SY, Yu R, Margolis RN, Evans RM, O’Malley BW. Evolution of NURSA, the Nuclear Receptor Signaling Atlas. Mol. Endocrinol. 2009; 23:740746. [PubMed: 19423650]

4. Carroll SM, Bridgham JT, Thornton JW. Evolution of hormone signaling in elasmobranchs by exploitation of promiscuous receptors. Mol. Biol. Evol. 2008; 25:2643-2652. [PubMed: 18799714]

5. Keay J, Thornton JW. Hormone-activated estrogen receptors in annelid invertebrates: Implications for evolution and endocrine disruption. Endocrinology. 2009; 150:1731-1738. [PubMed: 19036877]

6. LaFrate AL, Carlson KE, Katzenellenbogen JA. Bioorg. Med. Chem. 2009; 17:3528-3535. [PubMed: 19394231]

7. Ribeiro RC, Apriletti JW, Wagner RL, West BL, Fend W, Huber R, Kusher PJ, Hilsoon S, Scanlan T, Fletterick RJ, Schaufele F, Baxter JD. Mechanisms of thyroid hormone action: Insights from Xray crystallographic and functional studies. Recent Prog. Horm. Res. 1998; 53:351-392. [PubMed: 9769715]

8. Chandra V, Huang P, Hamuro Y, Raghuram S, Wang Y, Burris TP, Rastinejad F. Structure of the intact PPAR-gamma-RXR-alpha nuclear receptor complex on DNA. Nature. 2008; 456:350-356. [PubMed: 19043829]

9. Bisson WH, Abagyan R, Cavasotto CN. Molecular basis of agonicity and antagonicity in the androgen receptor studied by molecular dynamics simulations. J. Mol. Graph. Model. 2008; 27:452-458. [PubMed: 18805032]

10. Hassan AQ, Koh JT. Selective chemical rescue of a thyroid-hormone-receptor mutant, TRbeta(H435Y), identified in pituitary carcinoma and resistance to thyroid hormone. Angew. Chem. Int. Ed. Engl. 2008; 47:7280-7283. [PubMed: 18683837]

11. Venditti P, Chiellini G, Bari A, Di Stefano L, Zucchi R, Columbano A, Scanlan TS, Di Meo S. T3 and the thyroid hormone $\beta$-receptor agonist GC-1 differentially affect metabolic capacity and oxidative damage in rat tissues. J. Exp. Biol. 2009; 212:986-993. [PubMed: 19282495]

12. Meijsing SH, Pufall MA, So AY, Bates DL, Chen L, Yamamoto KR. DNA binding site sequence directs glucocorticoid receptor structure and activity. Science. 2009; 324:407-410. [PubMed: 19372434]

13. Marvin KA, Reinking JL, Lee AJ, Pardee KM, Krause HM, Burstyn JN. Nuclear receptors Homo sapiens Rev-erbbeta and Drosophila melanogaster E75 are thiolate-ligated heme proteins, which undergo redox-mediated ligand switching and bind CO and NO. Biochemistry. 2009; doi: 10.1021/ bi900697c

14. Madoux F, Li X, Chase P, Zastrow G, Cameron MD, Conkright JJ, Griffin PR, Thacher S, Hodder P. Potent, selective and cell penetrant inhibitors of SF-1 by functional ultra-high-throughput screening. Mol. Pharmacol. 2008; 73:1776-1784. [PubMed: 18334597]

15. Casey RJ, Desaulniers JP, Hojfeldt JW, Mapp AK. Expanding the repertoire of small molecule transcriptional activation domains. Bioorg. Med. Chem. 2009; 17:1034-1043. [PubMed: 18334300]

16. Han SJ, Lonard DM, O'Malley BW. Multi-modulation of nuclear receptor coactivators through posttranslational modifications. Trends Endocrinol. Metab. 2009; 20:8-15. [PubMed: 19019695] 
17. Campbell LA, Faivre EJ, Show MD, Ingraham JG, Flinders J, Gross JD, Ingraham HA. Decreased recognition of SUMO-sensitive target genes following modification of SF-1 (NR5A1). Mol. Cell. Biol. 2008; 28:7476-7486. [PubMed: 18838537]

18. Wu K, Katiyar S, Witkiewicz A, Li A, McCue P, Song LN, Tian L, Jin M, Pestell RG. The cell fate determination factor dachshund inhibits androgen receptor signaling and prostate cancer cellular growth. Cancer Res. 2009; 69:3347-3355. [PubMed: 19351840]

19. Gereben B, Zavacki AM, Ribich S, Kim BW, Huang SA, Simonides WS, Zeöld A, Bianco AC. Cellular and molecular basis of deiodinase-regulated thyroid hormone signaling. Endocr. Rev. 2008; 29:898-938. [PubMed: 18815314]

20. Arany Z, Wagner BK, Ma Y, Chinsomboon J, Laznik D, Spiegelman BM. Gene expression-based screening identifies microtubule inhibitors as inducers of PGC-1alpha and oxidative phosphorylation. Proc. Natl. Acad. Sci. U.S.A. 2008; 105:4721-4726. [PubMed: 18347329]

21. Wagner BK, Kitami T, Gilbert TJ, Peck D, Ramanathan A, Schreiber SL, Golub TR, Mootha VK. Large-scale chemical dissection of mitochondrial function. Nat. Biotechnol. 2008; 26:343-351. [PubMed: 18297058]

22. Norris JD, Joseph JD, Sherk AB, Juzumiene D, Turnbull PS, Rafferty SW, Cui H, Anderson E, Fan D, Dye DA, Deng X, Kazmin D, Chang CY, Willson TM, McDonnell DP. Differential presentation of protein interaction surfaces on the androgen receptor defines the pharmacological actions of bound ligands. Chem. Biol. 2009; 16:452-460. [PubMed: 19389631]

23. Pandey J, Koyutürk M, Kim Y, Szpankowski W, Subramaniam S, Grama A. Functional annotation of regulatory pathways. Bioinformatics. 2007; 23:i377-i386. [PubMed: 17646320]

24. Lin YS, Yasuda K, Assem M, Cline C, Barber J, Li CW, Kholodovych V, Ai N, Chen JD, Welsh WJ, Ekins S, Schuetz E. The major human PXR splice variant, PXR.2, exhibits significantly diminished ligand-activated transcriptional regulation. Drug Metab. Dispos. 2009; 37:1295-1304. [PubMed: 19251824]

25. Casas, C. Casals, Feige, JN., Desvergne, B. Interference of pollutants with PPARs: Endocrine disruption meets metabolism. Int. J. Obes. 2008; 32(suppl. 6):S53-S61.

26. Lee YK, Moore DD. Liver receptor homolog-1, an emerging metabolic modulator. Front Biosci. 2008; 13:5950-5958. [PubMed: 18508634]

27. Baxter JD, Webb P. Thyroid hormone mimetics: Potential applications in atherosclerosis, obesity and type 2 diabetes. Nat. Rev. Drug Discov. 2009; 8:308-320. DOI: 10.1126/scisignal.282mr5 [PubMed: 19337272] 


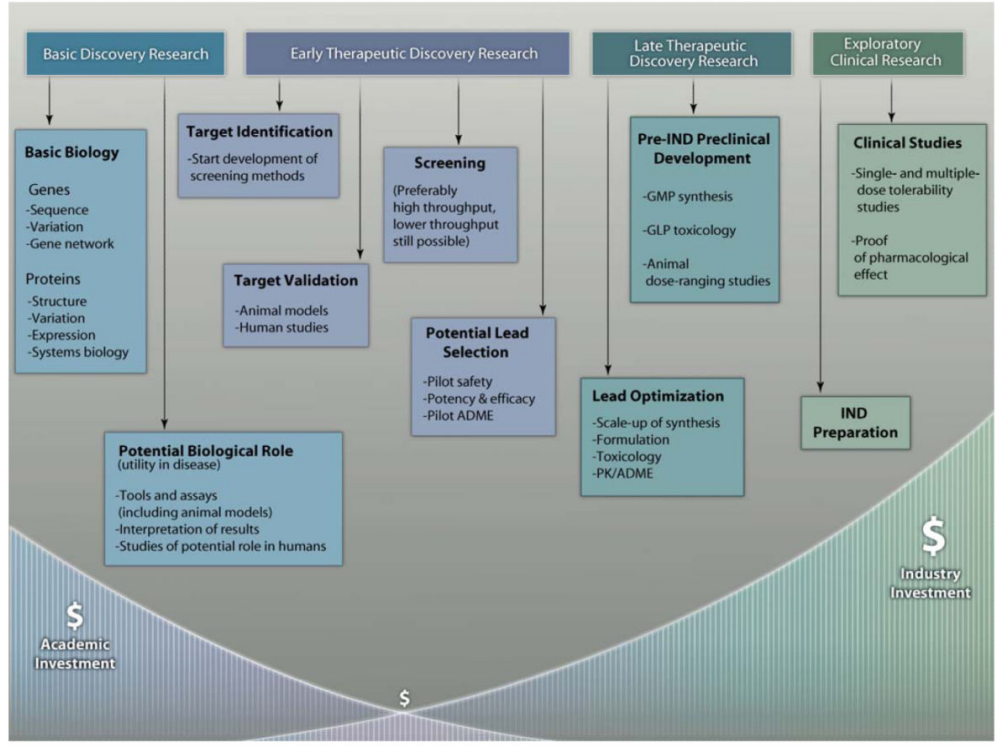

Fig. 1.

Pathway for translation of basic research to applications in the clinic. There is substantial investment from public and nonprofit institutions in basic discovery research and from the pharmaceutical industry in exploratory clinical research, but little investment in the translational research that moves potential therapeutic targets from the discovery phase toward clinical application. This is particularly true when it comes to pre-investigational new drug (Pre-IND) development. ADME, absorption distribution metabolism excretion; GMP, good manufactoring practice; GLP, good laboratory practice; and PK, pharmacokinetics. 